\title{
Study on Spectrum Estimation in Biophoton Emission Signal Analysis of Wheat Varieties
}

\author{
Yitao Liang, ${ }^{1,2}$ Hongxia Song, ${ }^{2}$ Qin Liu, ${ }^{3}$ Weiya Shi, ${ }^{1,2}$ and Lan $\mathrm{Li}^{1,2}$ \\ ${ }^{1}$ Key Laboratory of Food Information Processing and Control, Ministry of Education, Zhengzhou 450001, China \\ ${ }^{2}$ School of Information Science and Engineering, Henan University of Technology, Zhengzhou 450001, China \\ ${ }^{3}$ School of Electric and Information Engineering, Zhongyuan University of Technology, No. 41, Zhongyuan Road (M), \\ Zhengzhou 450007, China
}

Correspondence should be addressed to Yitao Liang; liangyt2011@126.com

Received 20 December 2013; Revised 17 May 2014; Accepted 21 May 2014; Published 17 June 2014

Academic Editor: Mingcong Deng

Copyright (C) 2014 Yitao Liang et al. This is an open access article distributed under the Creative Commons Attribution License, which permits unrestricted use, distribution, and reproduction in any medium, provided the original work is properly cited.

\begin{abstract}
The photon emission signal in visible range $(380 \mathrm{~nm}-630 \mathrm{~nm})$ was measured from various wheat kernels by means of a low noise photomultiplier system. To study the features of the photon emission signal, the spectrum estimation method of the photon emission signal is described for the first time. The biophoton emission signal, belonging to four varieties of wheat, is analyzed in time domain and frequency domain. It shows that the intensity of the biophoton emission signal for four varieties of wheat kernels is relatively weak and has dramatic changes over time. Mean and mean square value are obviously different in four varieties; the range was, respectively, 3.7837 and 74.8819. The difference of variance is not significant. The range is 1.1764 . The results of power spectrum estimation deduced that the biophoton emission signal is a low frequency signal, and its power spectrum is mostly distributed in the frequency less than $0.1 \mathrm{~Hz}$. Then three parameters, which are spectral edge frequency, spectral gravity frequency, and power spectral entropy, are adopted to explain the features of the kernels' spontaneous biophoton emission signal. It shows that the parameters of the spontaneous biophoton emission signal for different varieties of wheat are similar.
\end{abstract}

\section{Introduction}

Wheat is one of the food crops, which has the most widespread, the largest planting area, and the most trade. Varietal identification is not only the important content of germplasm resources, breeding research, and seed management but also the foundation of intellectual property and new variety protection $[1,2]$.

Currently, the methods of varietal identification are mainly morphology algorithm and biochemistry detection. Morphology algorithm is based on morphological characters to identify crop varieties. The method has drawbacks in long appraisal cycle, high cost, and low reliability [1]. Biochemistry test mainly contains protein electrophoresis analysis technology, zymogram technology, DNA molecular identification techniques, and so on. These methods can solve the problem of long morphological identification cycle, but there are some disadvantages in high analysis cost, poor repeatability, and high requirements in operation technology [2-5]. In addition, biochemical identification method belongs to the destructive detection and sometimes cannot meet the actual production requirements; rapid, nondestructive identification of wheat varieties has become an urgent problem to be solved.

With the development of technology, the biophotonics appeared. Biophoton emission can give overall information to describe the comprehensive factor of sample properties. Research shows that the biophoton emission exists in various biological systems and carries microinformation of biological systems. Biophoton emission was found to correlate with many biological processes and biological function. The experimental observation shows that different DNA conformations correspond to different intensity of biophoton emission [6]. Biophoton emission has high sensitivity to the change of internal biological system and the external environment. The detection and analysis in biophoton emission signal can obtain the information of internal biological system and display the microeffects of external environment. 


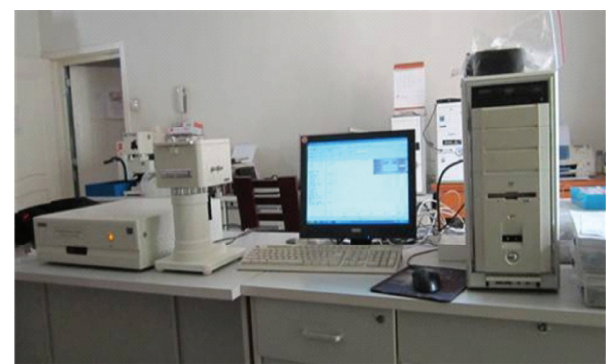

FiguRE 1: Picture of experiment measurement system.

However, there is an important theoretical and practical issue, which is how to establish an accurate biophoton characteristics model according to the change of biophoton emission signal. At present, the analytical methods of biophoton signal are only limited in time domain, which can only get the qualitative relations between biophoton emission signal and biophoton characteristics. The accurate model is difficult to get [7]. Therefore, for a more comprehensive study on the correlation between different wheat varieties and their biophoton emission signal, based on the theory of biophotonics, this paper tries to find the sensitive parameters to distinguish different wheat varieties by analyzing the biophoton emission signal in time domain and frequency domain and tries to look for a rapid and nondestructive method for identification of wheat varieties and then lay the foundation for the following model of variety identification.

\section{Materials and Methods}

2.1. Materials. Different varieties of wheat seeds, Zhengmai 7698 (Zhengmai for short), Yanzhan 4110 (Yanzhan), Wenmai 18 (Wenmai for short), and AK58 were harvested in June, 2012.

Select some different varieties of wheat seeds of full grain, wash wheat seeds 3 times in pure water, put in a drying box, dry to moisture content $(13.7 \pm 0.2) \%$, and then weigh wheat $5 \pm 0.02 \mathrm{~g}$ in each measurement.

\subsection{Measurement of Biophoton Emission. BPCL-ZL-TGC} ultraweak luminescence measuring instrument was used in the experimental measurement and experimental system as shown in Figure 1. Its detection sensitivity is $10^{-16} \mathrm{~W} /\left(\mathrm{s} \cdot \mathrm{cm}^{2}\right)$, and its detecting spectrum is $380 \mathrm{~nm}-630 \mathrm{~nm}$. The instrument is equipped with semiconductor refrigeration device, which can effectively reduce the detector dark noise. There is a significant difference between the noise and noise on preheating after 30 minutes. It is found that noise after preheating 30 minutes is stable, so the instrument should be preheated for 30 minutes before the measurements.

To magnify the electric field between the two electrodes of detector, high voltage is necessary and needs to be adjusted with standard radiation source (C-17). The top detecting efficiency can be got when the control voltage is supplied (about $1030 \mathrm{~V}$ ).

Biophoton emission signal of different wheat varieties is measured under standard conditions, which is determined by orthogonal experimental design $[8,9]$. Some uncertain factors, such as the change of condition, the disturbance of parameter, various failures, and other external disturbances, will have an effect on the system modeling. To ensure the accuracy and efficiency of experiment, it is necessary to have a certain experiment environment $[10,11]$.

Before measurement, samples of wheat are put in sample chamber for dark treatment for 30 minutes at the temperature of $28^{\circ} \mathrm{C}$. Noise is measured for 100 seconds before every sample measurement. Then the sample was measured for 1024 seconds, and mean of noise was directly subtracted by instrument during the sample measurement process. The interval time of sample measurement is set to $1 \mathrm{~s}$. The luminous intensity was recorded as photon number per unit time (counts/s). Different varieties were measured as 10 groups. The whole measuring process was in dark environment set by shading cloth, and the demand of the surrounding environment is that the temperature is $20 \pm 1^{\circ} \mathrm{C}$ when the humidity is $28 \pm 3 \% \mathrm{rh}$.

2.3. Analysis Method in Time Domain. Time domain analysis is that biophoton emission signal of wheat seed is regarded as the random function of time, and then analyze some statistical characteristics of the signal [12]. As a description for the biophoton emission signal in time domain, this paper uses statistical parameters as follows: the mean, variance, mean square value, and the correlation function.

Pretreatment: To get the array after denoising the experimental data based on the wavelet transform.

Define a random sequence of radiation signal from spontaneous photons of wheat seed, and its length is $N: x(n)=$ $\left\{x_{0}, x_{1}, x_{2}, \ldots, x_{i}, x_{i+1}, \ldots, x_{N-1}\right\}$. The average function is the cumulative average of the amplitude sequence of biophoton emission signal in the time coordinate as follows:

$$
m_{x}=E[x(n)]=\frac{1}{N} \sum_{i=0}^{N-1} x_{i} .
$$

The variance value can reflect the change tendency of difference between the average value and real-time value of radiation signal from spontaneous photons. Consider the following:

$$
\sigma_{x}^{2}(n)=E\left\{\left[x(n)-m_{x}\right]^{2}\right\}=\frac{1}{N} \sum_{i=0}^{N-1}\left(x_{i}-m_{x}\right)^{2} .
$$

RMS value can reflect the change of strength or energy of signal. Consider the following:

$$
E\left[x^{2}(n)\right]=\sigma_{x}^{2}(n)+m_{x}^{2} .
$$

The autocorrelation function can reflect the relationship of different values in different time. Two different random signals may have similar average value and variance value, but there may be an obvious difference between the two random signals. This difference can be described by correlation function. Consider the following:

$$
R(m)=\frac{1}{N} \sum_{i=0}^{N-|m|-1} x_{i} x_{i+m}, \quad m \in[0, N-1],
$$




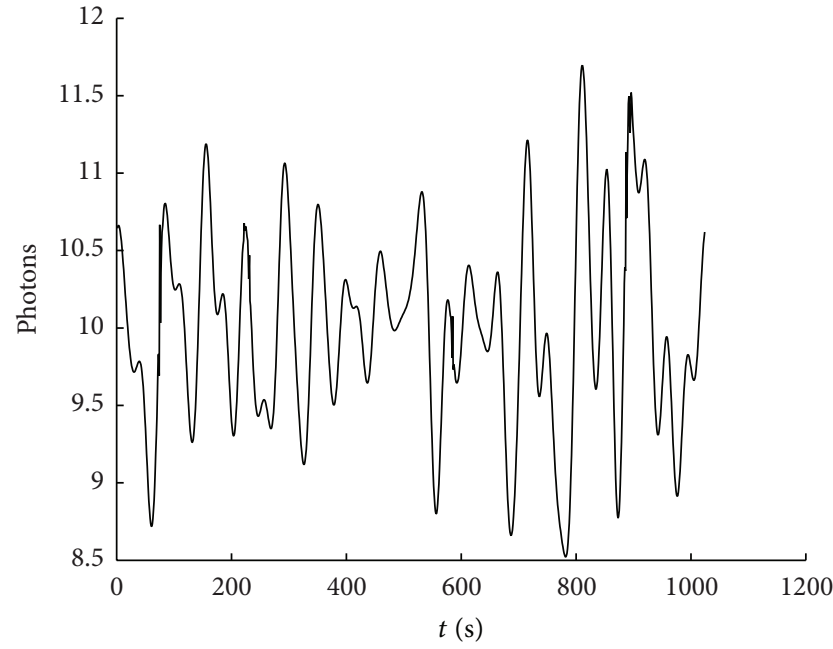

(a) Zhengmai

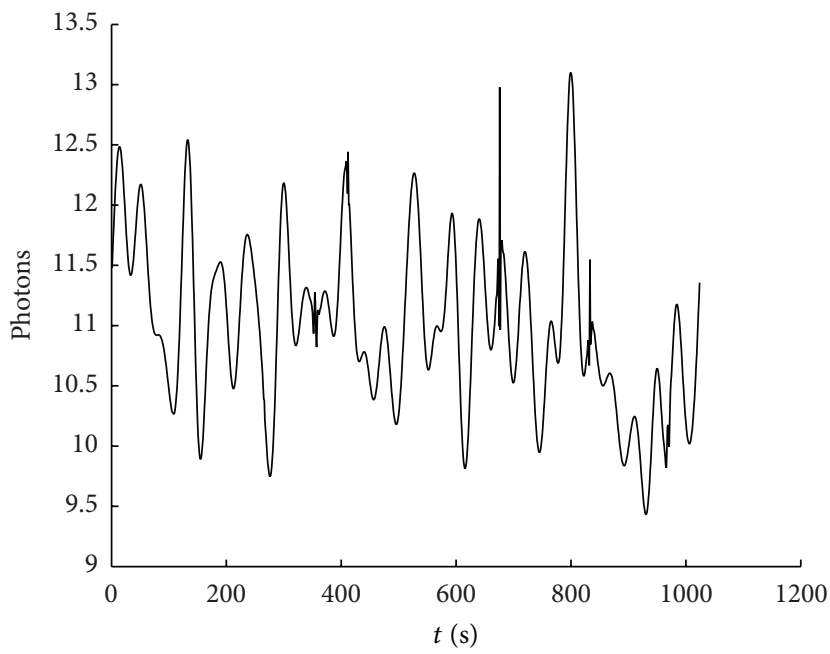

(c) Wenmai

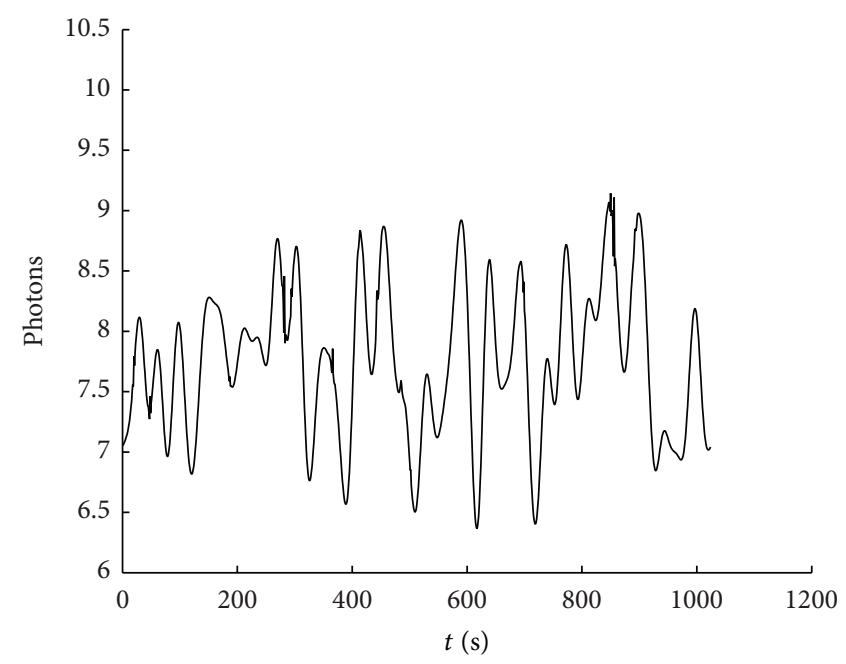

(b) Yanzhan

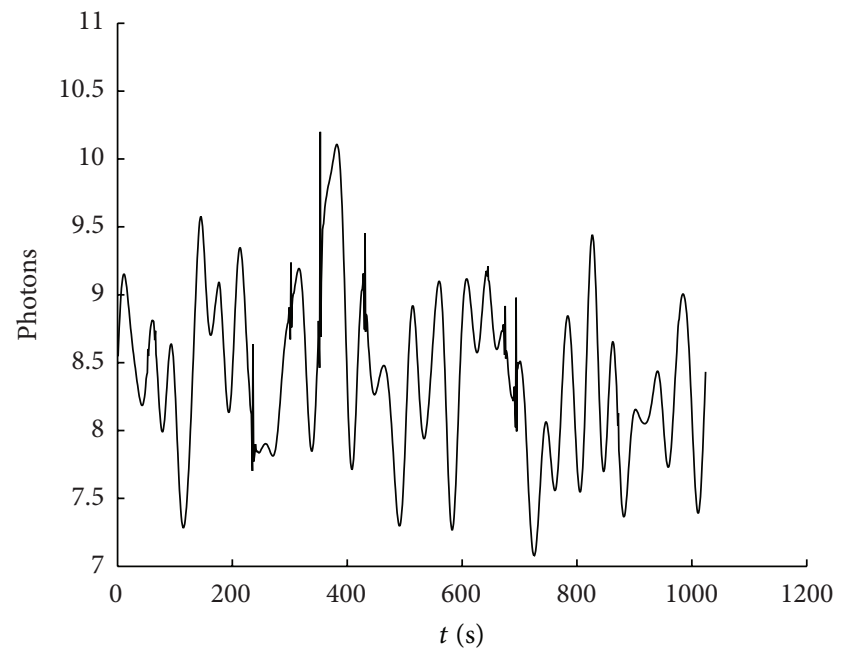

(d) Aikang

FIGURE 2: Waveforms of biophoton emission signal of four varieties in time domain.

where $m$ is a round figure, standing for the interval between two numbers. The equation of normalized correlation function $R_{\text {nac }}(m)$ is as follows:

$$
R_{\mathrm{nac}}(m)=\frac{R(m)}{R(0)}
$$

This method can decrease the effect of trend component [13].

2.4. Power Spectrum Density Analysis Method. Power spectrum density analysis is a main method to analyze random signal in frequency domain. This method can show the distributed characteristic of radiation signal in frequency domain. In this paper, classical spectral estimation method was chosen, and its theoretical foundation is WienerKhintchine theorem [14].
Random signal acquired from experiment $x(n)=$ $\left\{x_{0}, x_{1}, x_{2}, \ldots, x_{i}, x_{i+1}, \ldots, x_{N-1}\right\}$ needs to be treated by zeromean method.

Wiener-Khintchine theorem: wide power spectral density of stationary random process is the autocorrelation function of the Fourier transform.

Based on Wiener-Khintchine theorem and (4) and (5), we can get the equation of power spectrum density analysis:

$$
P_{\mathrm{BT}}(k)=\sum_{m=0}^{N-1} R_{\mathrm{nac}}(m) e^{-j 2 \pi(k / N) m} .
$$

We choose three characteristic parameters of power spectrum density analysis, as sensitive indicators, to find radiation characteristic from spontaneous photons of different types of wheat seeds [15].

(a) Spectral edge frequency (SEF): the signal power from $0 \mathrm{~Hz}$ to this frequency is 95 percent from total power, 


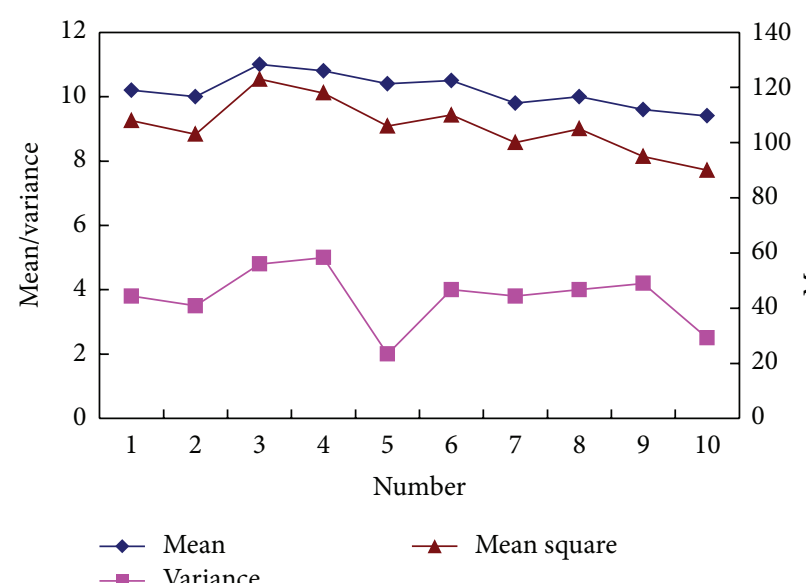

(a) Zhengmai

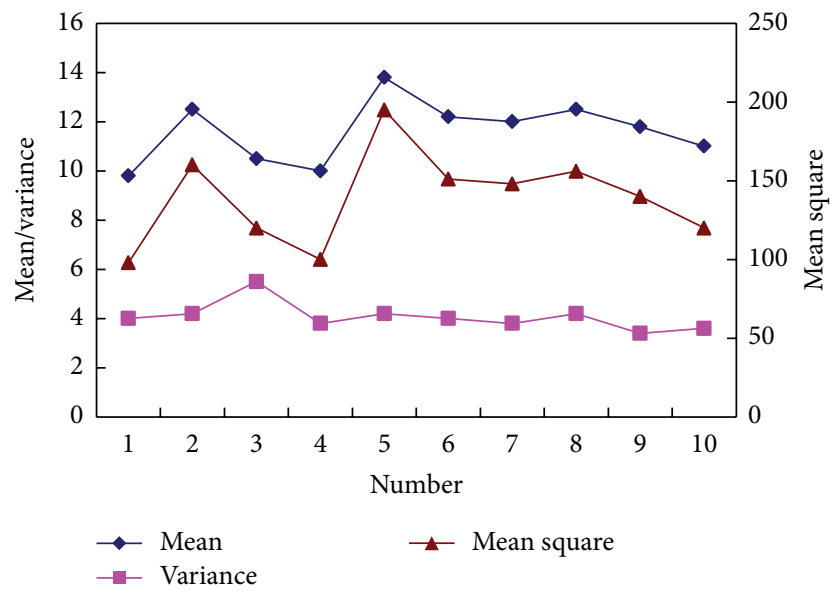

(c) Wenmai

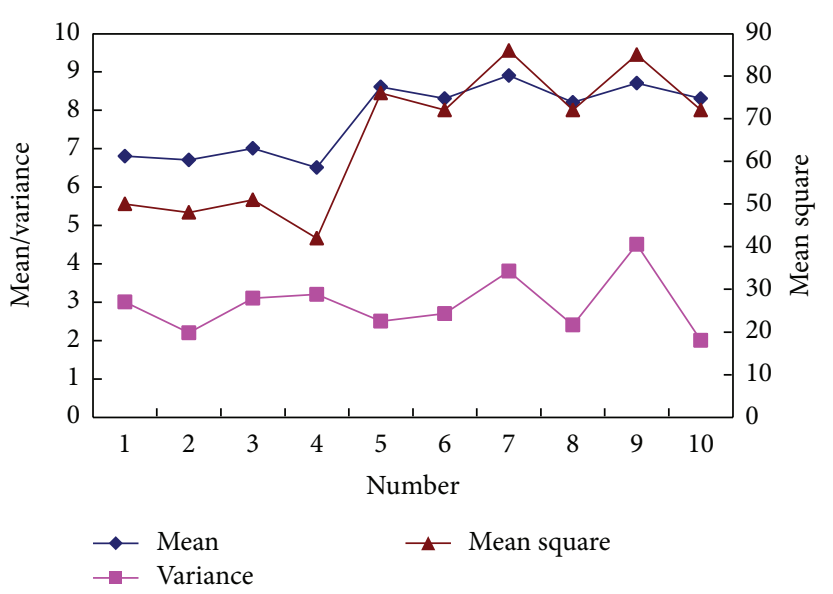

(b) Yanzhan

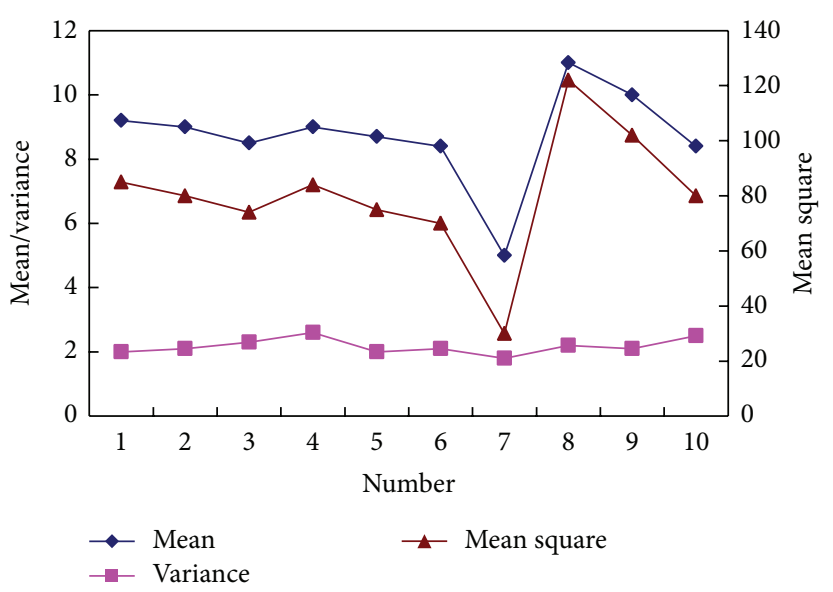

(d) Aikang

FIGURE 3: The statistical parameters distribution diagram of ten groups for four varieties.

and its unit is $\mathrm{Hz}$; SEF stands for the frequency range of the signal distribution.

(b) Spectral gravity frequency (SGF): this parameter can show the main distribution of signal in frequency domain. Its equation is as follows:

$$
f_{G}=\frac{\sum_{f=f_{s}}^{f_{e}}[P(f) \times f]}{\sum_{f=f_{s}}^{f_{e}} P(f)},
$$

where $f_{G}$ stands for the main frequency, the frequency range is between $f_{s}$ and $f_{e}$, and $P(f)$ stands for the power of signal.

(c) Power spectral entropy (PSE): this parameter can express the complexity of signal. Its definition $H_{f}$ is as follows:

$$
H_{f}=-\sum p_{f} \ln p_{f}
$$

While $f$ stands for frequency, $p_{f}$ means the energy percent.

\section{Analysis and Results}

The data of biophoton emission signal of four varieties were processed by using Matlab 7.1 software; the results are as follows. The paper intuitively analyzes biophoton emission signal of different varieties.

\subsection{Analysis of Biophoton Emission Signal of Four Varieties in} Time Domain. Waveforms of biophoton emission signal of each variety in time domain were shown in Figure 2. The data of each variety was the mean of ten groups of independent repeated experiments after wavelet denoising. Intuitively, the fluctuation of biophoton emission signal of wheat has no evident regularity and is nondeterministic functions of time, that is, random signal. Different varieties, the fluctuation also has the difference.

3.2. Statistical Parameters. The statistical parameters of biophoton emission signal of four varieties for ten groups of repeated experiment are shown in Figure 3.

As shown in Figure 3, the mean, variance, and mean square of ten groups of repeated experiment for different 


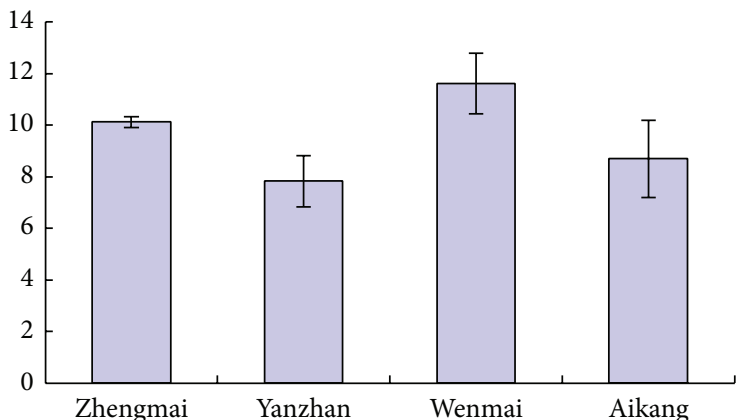

$\square$ Mean

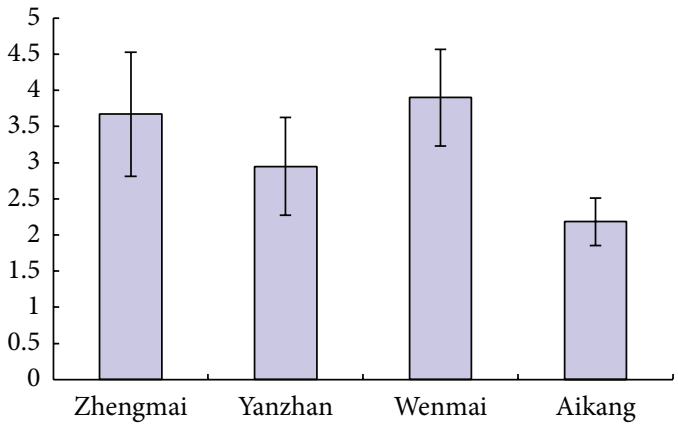

Variance

(a) Mean

(b) Variance

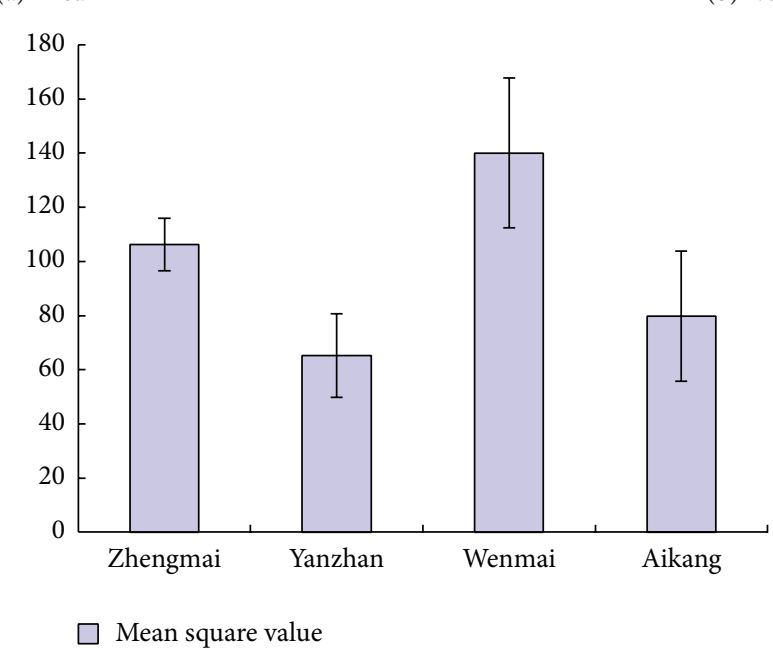

(c) Mean square value

FIGURE 4: The statistical parameters of biophoton emission signal of four varieties.

varieties were fluctuating within a certain range, on average. Mean value of four varieties was, respectively, around 10, 8, 12, and 9 , and the variance was around $4,3,4$, and 2 , with smaller fluctuation. Mean square values were around 100, 70, 140, and 80 , with greater fluctuation.

Statistical parameters of four varieties were, respectively, averaged, and results were shown in Table 1.

As shown in Figure 4 and Table 1, for mean and mean square value, there were significant differences between four varieties, and the range was, respectively, 3.7837 and 4.8819. There were greater values for Zhengmai and Wenmai. For the variance, there were no significant differences between four varieties, and the range is only 1.7164. Two statistical parameters of the mean and mean square value have a strong correlation, and the characteristics of the overall biophoton emission signal are mostly positive after denoising.

The mean and the mean square value represent the amplitude of characteristic, and there were great differences in amplitude between four varieties. So we can use the amplitude of differential varieties as the characteristic parameter to identify the varieties.
3.3. Autocorrelation. By analyzing autocorrelation function graph of biophoton emission signal of four wheat varieties, we found that the maximum of correlation function is in $m=0$, along with the change of absolute value of $m$ of the value of correlation function changes, which shows that biophoton emission signal of wheat changes greatly with time varying (Figure 5).

From the analysis in the statistical parameters, we found that the variance of Wenmai and Zhengmai is relatively close. In the correlation function, the ratio of the first peak and second peak is also relatively close. According to the analysis results, they show that the variance and correlation function cannot distinguish between different varieties well.

3.4. Analysis of Power Spectrum. As shown in Figure 6, it is the power spectrum simulation curve of biophoton emission signal of four varieties.

As shown in Figure 6, power spectrum of biophoton emission signal of four varieties is mainly concentrated in the band from 0 to 0.1 . Enveloping part by the dotted line 


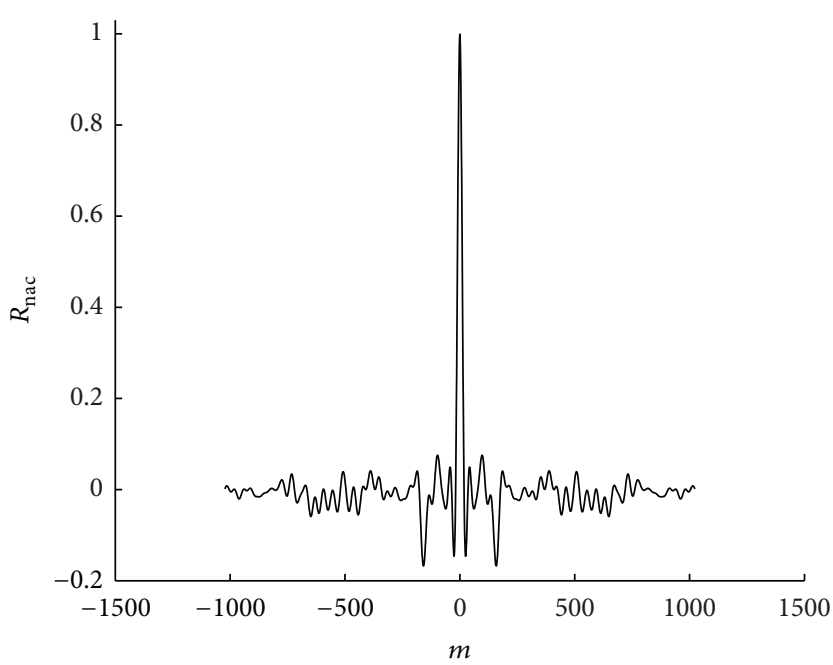

(a) Zhengmai

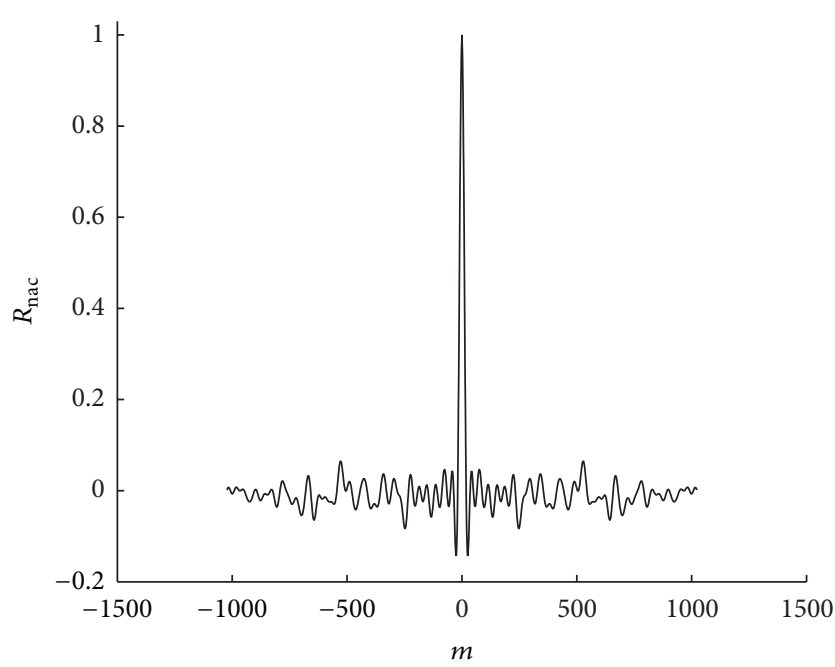

(c) Wenmai

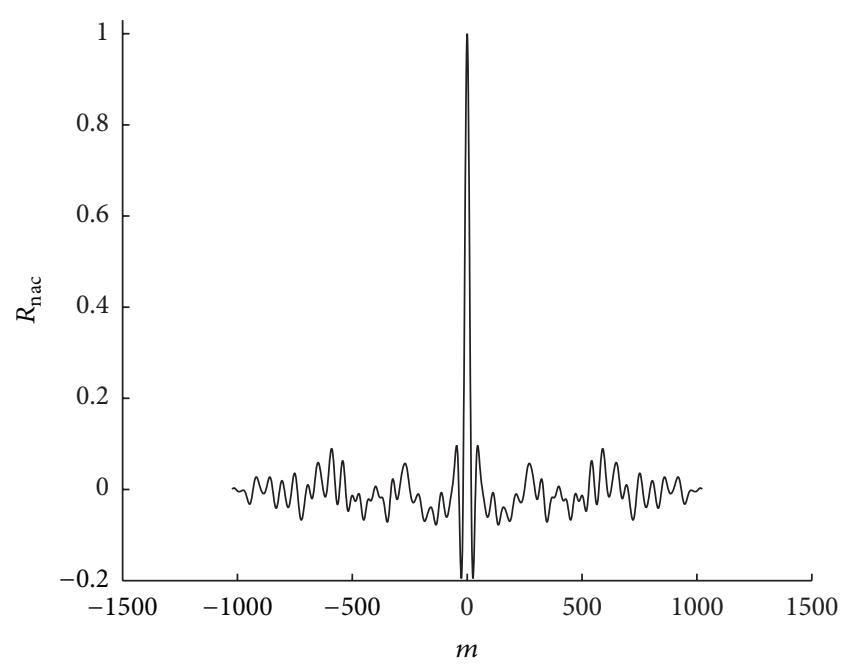

(b) Yanzhan

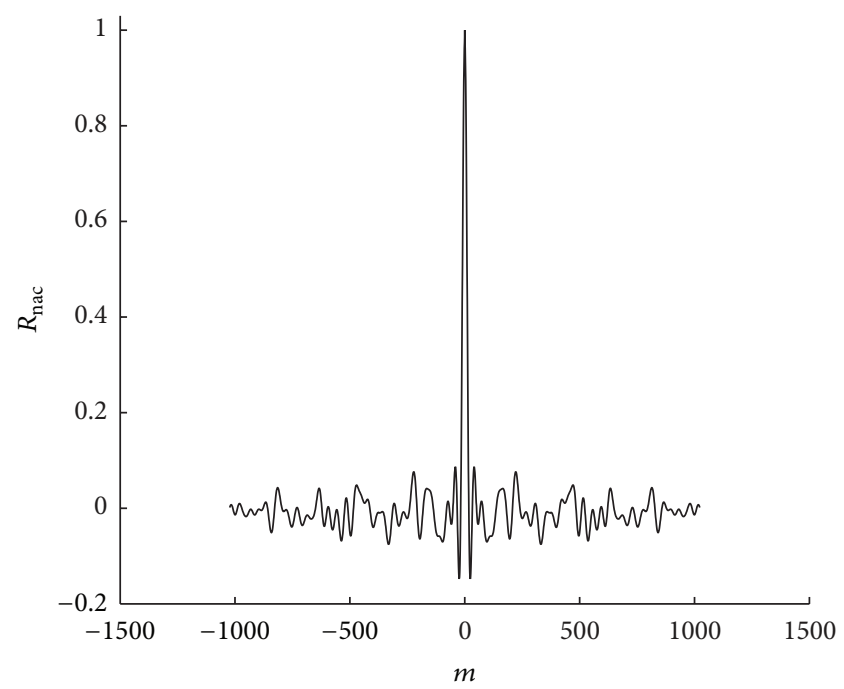

(d) Aikang

FIGURE 5: Normalized autocorrelation function graph of biophoton emission signal of four varieties.

TABLE 1: The statistical parameters of biophoton emission signal of four varieties.

\begin{tabular}{lcccc}
\hline & Zhengmai & Yanzhan & Wenmai & Aikang \\
\hline Mean & $10.1177 \pm 0.2077$ & $7.8308 \pm 0.9967$ & $11.6145 \pm 1.1834$ & $8.6961 \pm 1.4961$ \\
Variety & $3.6695 \pm 0.8605$ & $2.9481 \pm 0.6754$ & $3.8998 \pm 0.6686$ & $2.1834 \pm 0.3267$ \\
Mean square value & $106.2239 \pm 9.7302$ & $65.1631 \pm 15.4674$ & $140.055 \pm 27.6494$ & $79.8201 \pm 23.9980$ \\
\hline
\end{tabular}

shows that there were obvious crests in the band from 0 to 0.1 . For varieties of Zhengmai and Wenmai, there is a crest in this band, while there are two crests for Aikang and Yanzhan. We also found that the curve of the power spectrum is flat, which has the similar frequency domain characteristics to white noise. According to the amplitude of peak point, we can know that the values of Yanzhan and Aikang are higher than Zhengmai and Wenmai, and the range is 4.08 .
Calculate SEF, SGF, and PSE of ten groups of four varieties, respectively, and then average the parameters; the results were shown in Table 2.

As shown in Figure 7 and Table 2, SEF of four varieties is close and located at around $0.4 \mathrm{~Hz}$, and the range is 0.00409 . SGF of four varieties is also close, and the range is 0.00242 , with the similar tendency to SEF. For PSE, there are greater differences among four varieties, in which PSE of Yanzhan is maximal; its range is 0.0205 . From the curve of the power 


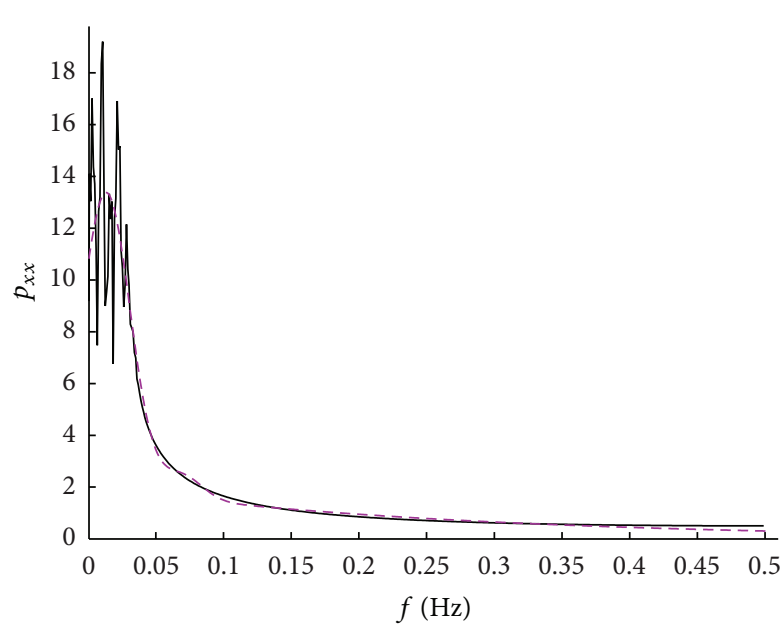

(a) Zhengmai

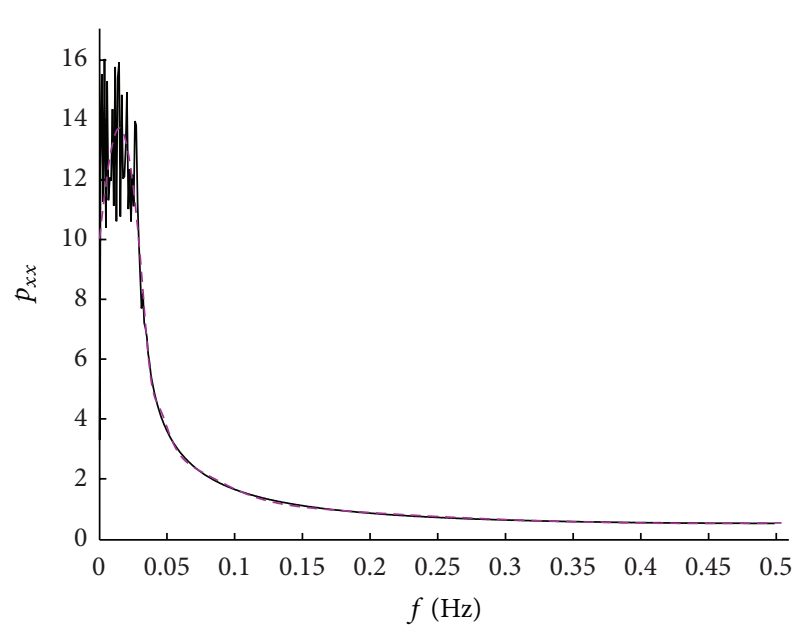

(c) Wenmai

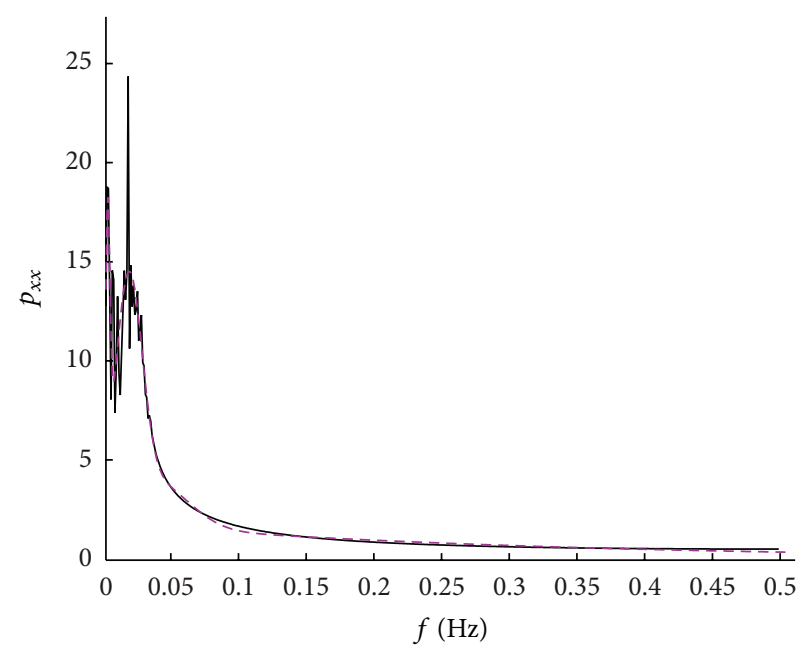

(b) Yanzhan

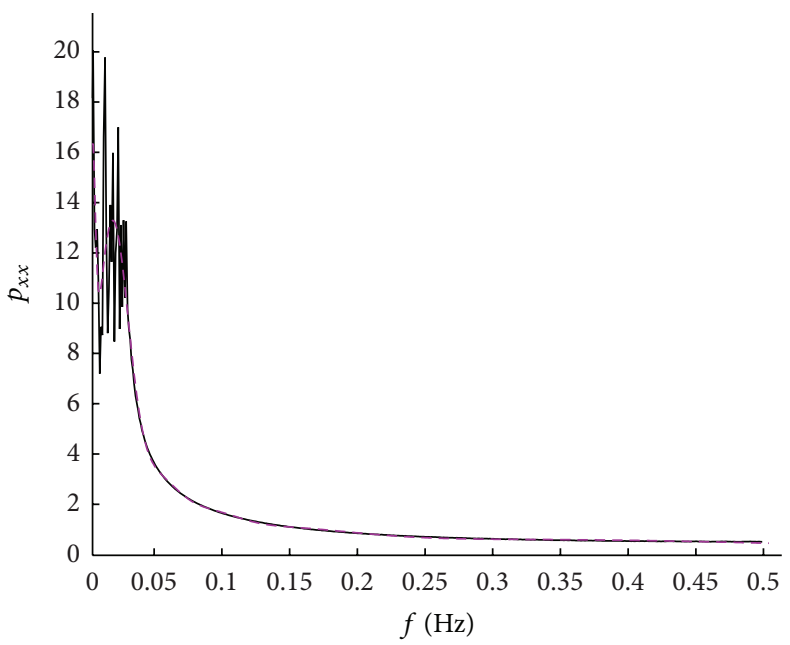

(d) Aikang

FIGURE 6: Envelope of curve of power spectrum of biophoton emission signal of four varieties.

TABLE 2: Parameters of power spectrum.

\begin{tabular}{lcccr}
\hline & Zhengmai & Yanzhan & Wenmai & Aikang \\
\hline SEF $(\mathrm{Hz})$ & $0.40959 \pm 0.0015$ & $0.41368 \pm 0.0092$ & $0.41085 \pm 0.0051$ & $0.41281 \pm 0.0046$ \\
SGF $(\mathrm{Hz})$ & $0.10567 \pm 0.0018$ & $0.10809 \pm 0.0067$ & $0.10639 \pm 0.0028$ & $0.10684 \pm 0.0022$ \\
PSE & $5.42725 \pm 0.0351$ & $5.44775 \pm 0.0695$ & $5.4323 \pm 0.0489$ & $5.42898 \pm 0.0436$ \\
\hline
\end{tabular}

spectrum of four varieties, we can also find that the curve of power spectrum of Yanzhan is the narrowest, and the curve becomes relatively large with the magnitude of change.

\section{Conclusion}

Biophoton emission signal can reflect general information of biology. In time domain, we found the intensity of light from four different types of wheat was relatively low. The average and variance of four types of wheat were obviously different; the variation was 7.387 and 74.8926 individually. However, the RMS value and correlation coefficient had small differences.
The variation was 1.4861 and 0.72 individually. In frequency domain, the peak numbers of power curves from four types of wheat were different; central frequency could not be found, and hidden message could be gotten due to the limit of the classical spectral estimation method. This limitation would be overcome in the following research. Besides, the four types of wheat had similar power spectrum parameters. So the wheat varieties cannot be distinguished by these three chosen characteristic parameters well.

Based on analysis in time and frequency domain, average value, number and scope of peak, and variance value proved to be suitable characteristic parameters. For example, Zhengmai or Wenmai often had big average value and variance 


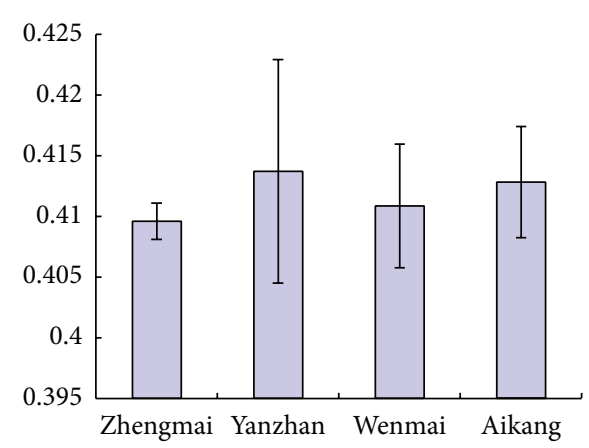

$\operatorname{SEF}(\mathrm{Hz})$

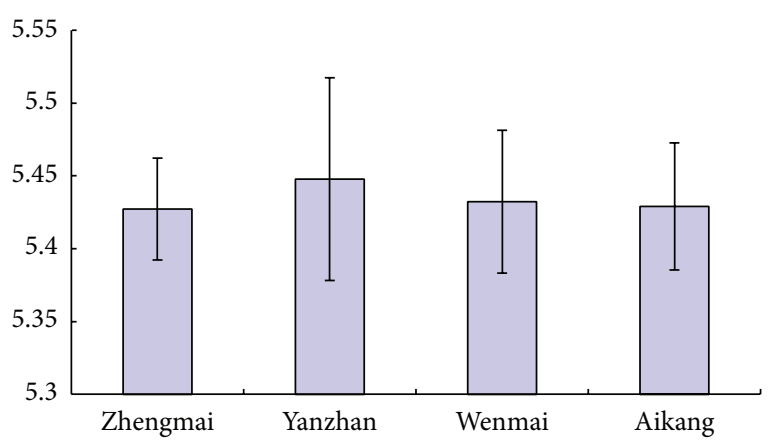

SGF $(\mathrm{Hz})$

(a) SEF

(b) SGF

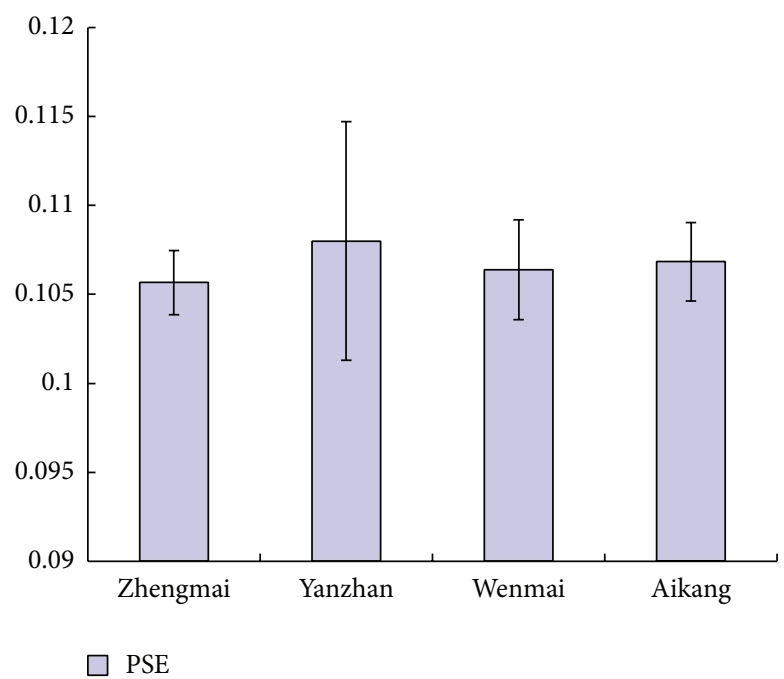

(c) PSE

FIGURE 7: Parameters of power spectrum of biophoton emission signal of four varieties.

value; the peak value of frequency spectrum could be used to distinguish Zhengmai and Wenmai exactly. To improve the accuracy, individual data base of different wheat and more characteristic parameters could also be built and chosen.

In this paper, a method of the time frequency analysis was used to recognize the types of wheat by analyzing the signal of biological spontaneous photon radiation. The experiment proved that this method is feasible, and it is a new method to identify different types of wheat.

\section{Conflict of Interests}

The authors declare that there is no conflict of interests regarding the publication of this paper.

\section{Acknowledgments}

This work is sponsored by National Natural Science Foundation of China (31171775, 11201120, 61174056, and 61201389) and National High Technology Research and Development Program of China (2012AA101608) and supported by
Province Technology R \& D Program of Henan Province of China (142102210148). Research project was supported by the Key Laboratory of Grain Information Processing and Control.

\section{References}

[1] Y. Yueming and L. Guangming, "Studies on capillary electrophresis separation of wheat gliadins and use in vaietal identification," Acta Agronomica Sinica, vol. 25, no. 2, pp. 241243, 1999.

[2] L. Li, C. Xinmin, and H. Zhonghu, "Application of capillary electrophresis in varietal identification and quality," Journal of Triticeae Crops, vol. 27, no. 2, pp. 229-236, 2007.

[3] W. Xiaoyan, Z. Huarong, C. Guanha, T. Yiling, and W. Shumin, "Study on the identification of wheat varieties by capillary zone electrophoresis," Journal of Agricultural University of Hebei, vol. 26, no. 2, pp. 10-12, 2003.

[4] Y. Yan, G. Liu, S. Prodanovic, G. Surlan-Momirovic, D. Zoric, and D. Perovic, "Separation of wheat glutenin subunits and varietal identification," Journal of the Chinese Cereals and Oils Association, vol. 13, no. 6, pp. 1-5, 1998. 
[5] R. Y. Li, Q. Li, W. L. Zhang, H. Zhang, G. A. Song, and D. J. Wang, "Application of SSR markers to identification and protection of wheat variety," Shandong Agricultural Sciences, vol. 6, no. 2, pp. 14-17, 2007.

[6] G. Qiao, Biophotonics, Sciences Press, Beijing, China, 2007.

[7] S. Wen, M. Deng, S. Bi, and D. Wang, "Operator-based robust nonlinear control and its realization for a multi-tank process by using a distributed control system," Transactions of the Institute of Measurement and Control, vol. 34, no. 7, pp. 891-902, 2012.

[8] P. Rui, Research on spontaneous biophoton characteristics of wheat kernels with hidden insects [M.S. thesis], Henan University of Technology, 2013.

[9] Z. Yuankun, Analysis of the delayed luminescence of the wheat kernel and hidded insect [M.S. thesis], Henan University of Technology, 2013.

[10] S. Wen and M. Deng, "Operator-based robust nonlinear control and fault detection for a Peltier actuated thermal process," Mathematical and Computer Modelling, vol. 57, no. 1-2, pp. 1629, 2013

[11] N. Bu and M. Deng, "System design for nonlinear plants using operator-based robust right coprime factorization and isomorphism," IEEE Transactions on Automatic Control, vol. 56, no. 4, pp. 952-957, 2011.

[12] L. Wang, H. Li, M. Lin, Q. Li, F. Lou, and J. Chen, "Analysis of plant electrical signal in the time domain and frequency domain," Journal of China Jiliang University, vol. 16, no. 4, pp. 411-416, 2011.

[13] Y. Lvxi, Modern Digital Signal Processing, Sciences Press, Beijing, China, 2008.

[14] L. Ying, Digital Signal Processing, Publishing House of electronics industry, Beijing, China, 2004.

[15] Z. Xiaohui, Y. Ningmei, and X. Gang, "The analysis on spectrum characteristic of plant electrical signal based on wavelet denoising," Journal of Xian University of Technology, vol. 27, no. 4, pp. 411-441, 2011. 


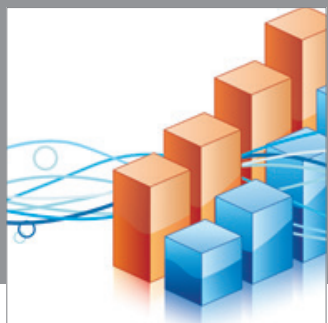

Advances in

Operations Research

mansans

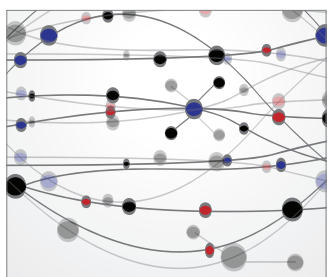

The Scientific World Journal
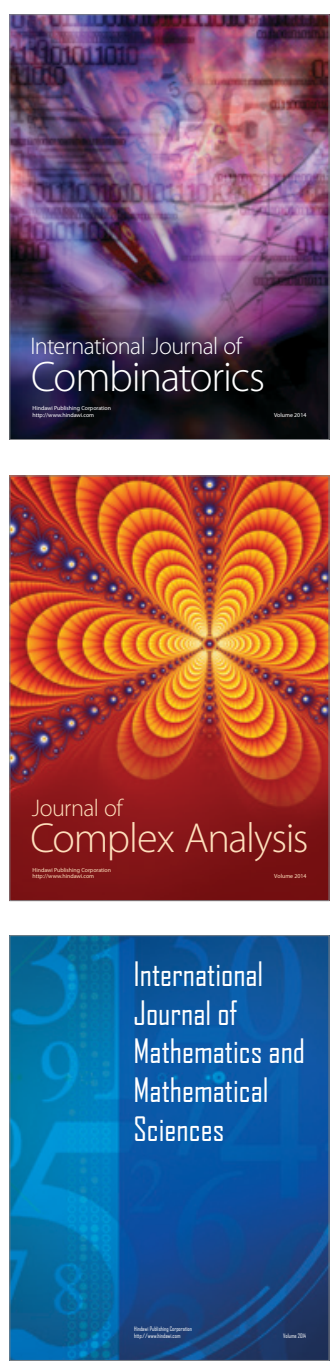
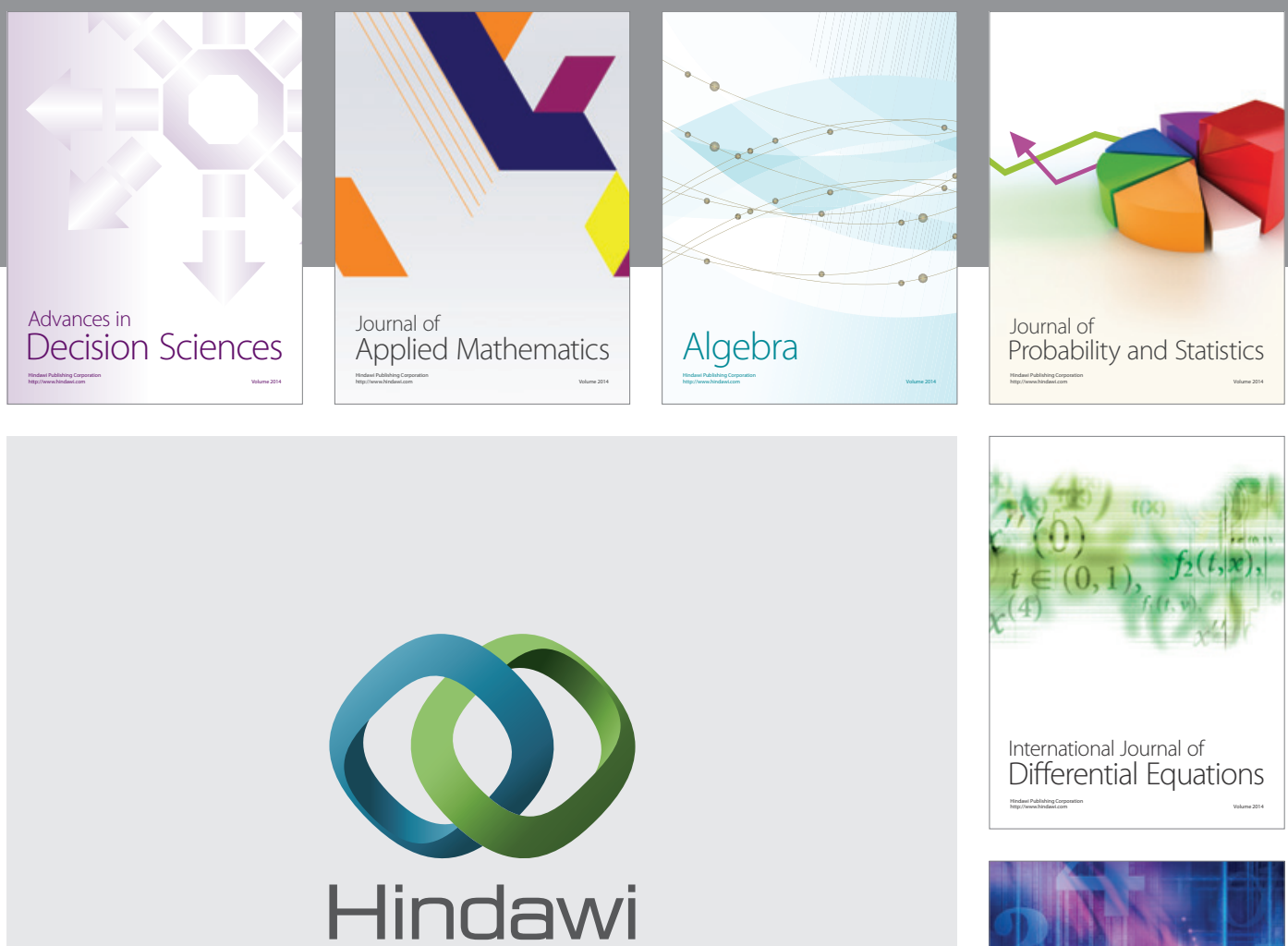

Submit your manuscripts at http://www.hindawi.com
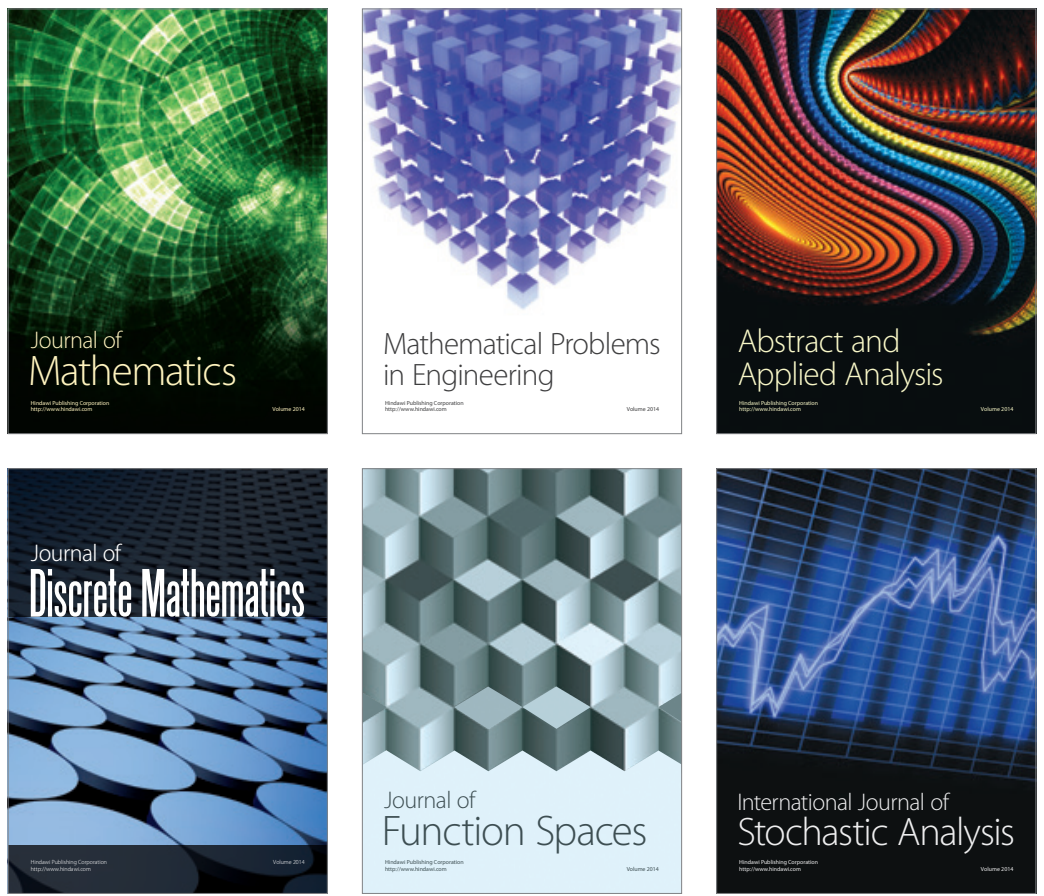

Journal of

Function Spaces

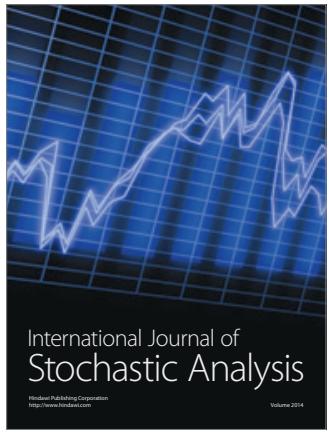

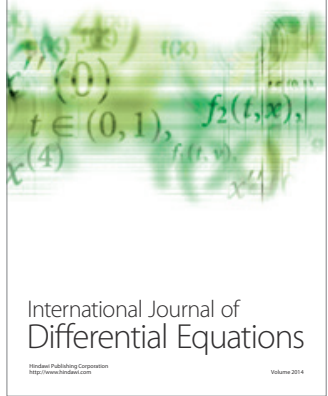
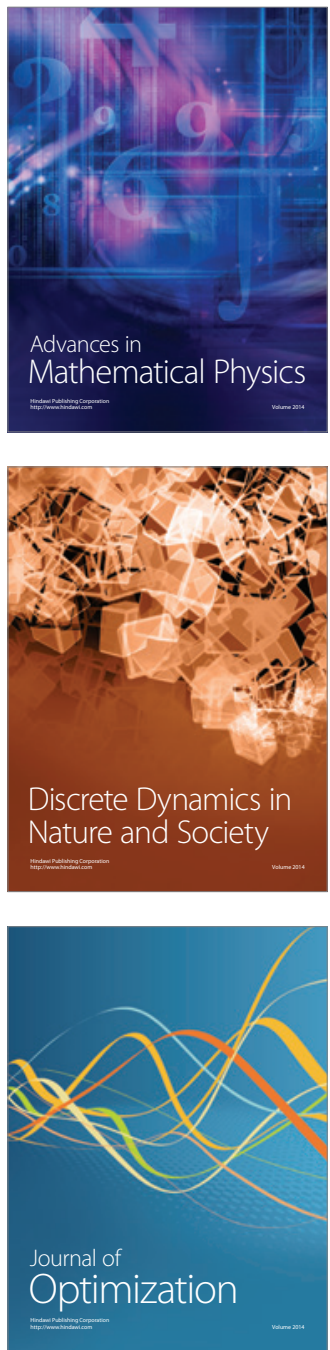